\title{
Experimental Demonstration of Coexistence of Microwave Wireless Communication and Power Transfer Technologies for Battery-Free Sensor Network Systems
}

\author{
Satoshi Yoshida, ${ }^{1}$ Takumasa Noji, ${ }^{2}$ Goh Fukuda, ${ }^{3}$ Yuta Kobayashi, ${ }^{1}$ and Shigeo Kawasaki ${ }^{1}$ \\ ${ }^{1}$ Institute of Space and Astronautical Science, Japan Aerospace Exploration Agency, 3-1-1 Yoshinodai, \\ Chuo, Sagamihara, Kanagawa 252-5210, Japan \\ ${ }^{2}$ Tokyo Metropolitan University, 1-1 Minami-Osawa, Hachioji, Tokyo 192-0397, Japan \\ ${ }^{3}$ Tokyo University of Science, 1-14-6 Kudankita, Chiyoda-ku, Tokyo 102-0073, Japan
}

Correspondence should be addressed to Satoshi Yoshida; yoshida.satoshi@jaxa.jp

Received 2 May 2013; Revised 24 July 2013; Accepted 4 September 2013

Academic Editor: Stavros Georgakopoulos

Copyright (C) 2013 Satoshi Yoshida et al. This is an open access article distributed under the Creative Commons Attribution License, which permits unrestricted use, distribution, and reproduction in any medium, provided the original work is properly cited.

\begin{abstract}
This paper describes experimental demonstrations of a wireless power transfer system equipped with a microwave band communication function. Battery charging using the system is described to evaluate the possibility of the coexistence of both wireless power transfer and communication functions in the C-band. A battery-free wireless sensor network system is demonstrated, and a high-power rectifier for the system is also designed and evaluated in the S-band. We have confirmed that microwave wireless power transfer can coexist with communication function.
\end{abstract}

\section{Introduction}

Most electrical devices are powered by wires, but wired connections limit the mobility of portable devices and the payloads of cars and spacecraft. There is thus a strong demand for practical wireless technologies to reduce the weight of communication and power-supply wiring. There are three wireless power transfer (WPT) categories: electromagnetic coupling type $[1,2]$, magnetic resonance type $[3,4]$, and microwave radiation type $[5,6]$. Table 1 summarizes important features of each category. Coupling- and resonant-type WPT devices using MHz frequencies have been reported [7, 8], but such devices are not suitable for high-speed signal and command transfer or cannot transfer energy over distances of several meters. Microwave WPT shows more promise, since the microwave band is commonly used in applications such as wireless local area network and cellular network systems. Also, WPT-based microwave bands can transfer both energy and command signals over long distances.

The WPT systems using microwave or millimeter wave bands are categorized into three types, according to power level. The first is the scavenging type, for targeting very weak power densities [9-11] such as in wide coverage services, for example, TV or radio broadcasting and cellular base stations $[6,12]$. The second is an energy harvester type, for targeting weak power densities [13] such as narrow coverage area services, for example, wireless local or personal area networks, and other mobile terminals. The third is active energy transfer systems such as those used in space solar power stations $[14,15]$ and wireless charging of electric vehicles $[5,16]$. Other energy sources such as heat and vibration can also be harvested [17-19]. Table 2 summarizes these categories. Microwave rectifiers are a key device for realizing these systems, in particular diode-based microwave rectifiers [2023]. These systems and rectifiers support only microwave WPT functions and do not coexist with communication functions.

We previously proposed a 4-bit digital phase shifter [24] and active integrated phased array antenna with beam steering capability [25] for effective use of microwave energy, since propagation loss is large compared to other WPT configurations. 
TABLE 1: WPT classifications by principle.

\begin{tabular}{lccc}
\hline Type & Range & Power delivery & Functionality \\
\hline EM coupling & Short & High & Communication \\
Magnetic resonance & Medium & High/medium & Power \\
Microwave power transmission & Long & Medium/low & Communication, sensors, and power \\
\hline
\end{tabular}

TABLE 2: WPT classifications by power level.

\begin{tabular}{lccc}
\hline Type & Source & Available transferred power & Structure \\
\hline Scavenging & Unknown (RF and mechanical) & $\mu \mathrm{W}$ & Simple \\
Harvesting & Intentional and unknown (RF) & $\mathrm{mW}$ & $\mathrm{W}$ \\
EM power receiving & Intentional (pair with RF power & transmission) & Complex \\
\hline
\end{tabular}

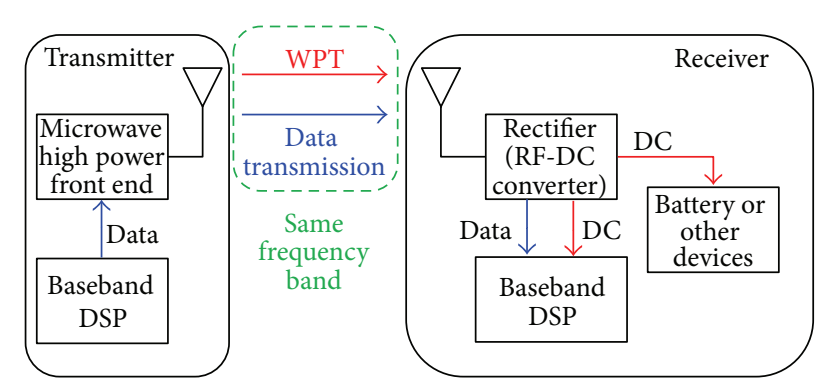

FIGURE 1: A conceptual illustration of WiCoPT technology using the microwave band.

This paper describes and discusses experimental demonstrations of wireless powering with communication technology using microwave bands. Section 2 introduces two concepts of wireless powering technologies. Section 3 designs and evaluates a microwave rectifier suitable for the technology and demonstrates a battery charging system using WPT integrated with communication technology and beam steering functions. Section 4 designs and evaluates a full wireless and battery-free sensor network system.

\section{Proposal and Design of WiCoPT and WiSEnT}

The main objective of our research group is to develop a completely wireless sensor network system for use in reusable rockets. Toward this end, we have proposed wireless information/communication and power transmission (WiCoPT) and wireless sensor and energy transfer (WiSEnT) technologies. Simultaneous wireless power and data transfer at microwave frequencies has been achieved in RFID applications. The differences between our proposal and RFID systems are the delivered power, frequency band, and operating time width. Practical RFID applications use milliwatt-class power levels, several tens of $\mathrm{MHz}$ frequency bands, and millisecond-order communication times, whereas our proposal uses watt-class power levels, microwave frequency bands, and communication/WPT time widths on the order of minutes or hours.

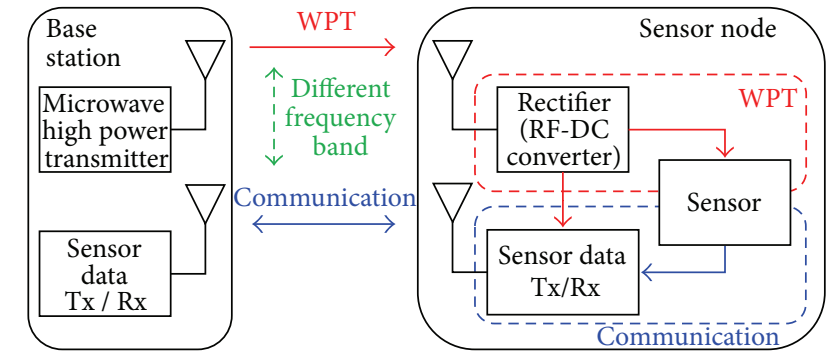

FIGURE 2: A conceptual illustration of WiSEnT technology using the microwave band.

Figure 1 shows a conceptual illustration of using WiCoPT technology for wireless power and communication functions. The same frequency band is effectively used for both WPT and communication, since the WPT system utilizes the $\mathrm{RF}$ power of the modulated signal. To eliminate the local oscillator (LO) source on the receiver side, the transmitter delivers the continuous wave (CW) signal, which is generated, amplified, and combined with the modulated signal in the microwave high-power front-end block. There are two methods for obtaining both DC power and intermediate frequency (IF) signals:

(1) use of a directional coupler before the rectifier to separate DC and IF,

(2) use of a band-pass filter (BPF) after the rectifier to separate DC and IF.

Sections 3.2 and 3.3, respectively, show examples of methods 1 and 2 . The generated DC power charges batteries or serves as a power supply for other devices. In method 1 , the rectifier in the receiver operates solely as an RF-DC converter. In method 2, the rectifier acts as both a downconvert mixer and an RF-DC converter. IF signals of the frequency difference between the modulated RF and nonmodulated LO signal from the transmitter are used for communication function.

Figure 2 shows a conceptual illustration of the WiSEnT technology, which is derived or produced from conventional wireless sensor network systems. The main difference 


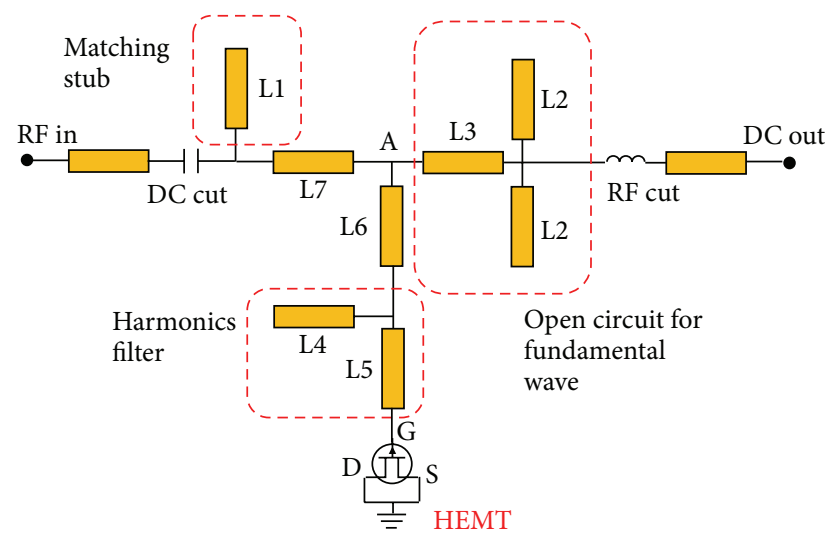

FIgURE 3: Circuit diagram of a basic design of the C-band rectifier using HEMT device.

between the WiSEnT technology and conventional wireless sensor networks is whether DC power is wireless or wired. The base station has a microwave-band WPT transmitter and communication transceiver for sensor control and data receiving, and the sensor node has a microwave-band rectifier that supplies DC power to sensor nodes. The sensor and data transceiver operate using DC power and thus do not require batteries. The WPT and communication signal frequencies are sufficiently different to avoid mutual interference. The illustration shows only one sensor node, but more nodes will be added in actual applications. The WiSEnT technology thus realizes a completely wireless sensor network.

\section{Experimental Demonstration of WiCoPT}

This section shows the microwave rectifier design and describes potential integration with beam steering technology and a battery charging demonstration of the WiCoPT technology.

3.1. Design and Evaluation of the Microwave Rectifier for WiCoPT. A Schottky diode is commonly used for microwave rectifiers. The rectifier converts microwave energy to DC energy used as a power supply for electrical or mechanical devices or for battery charging. We use a field-effect transistor, in particular a high-electron-mobility transistor (HEMT), as a rectifying device. The rectifier utilizes the HEMT as a diode when the drain and source of the HEMT terminals are grounded. The HEMT-type rectifier is easily integrated into a monolithic microwave integrated circuit for future miniaturization.

Figure 3 shows a circuit diagram for a basic design of the C-band rectifier using a HEMT device. The rectifier design method is based on configuration with an input/output filter [26]. Open stubs using a $50 \Omega$ microstrip line are used to configure the role of the input/output filter. A DC cut capacitor and an RF cut inductor are placed at the input and output port, respectively. The open stub near the DC cut capacitor is an input matching network to the RF connector. L2 and L3 have an initial length of $\lambda / 4$, since impedance from point $\mathrm{A}$ is open for a fundamental wave of $5.80 \mathrm{GHz}$ to flow the fundamental signal for the HEMT. L4 and L5, respectively, have an initial length of $\lambda / 8$ and $\lambda / 4$ as a filter with ideal impedance of 0 and infinite for odd- and evenorder harmonics.

Figure 4 shows pictures of fabricated rectifiers. C-band rectifiers without and with a BPF are shown in Figures 4(a) and $4(\mathrm{~b})$, respectively. The BPF is integrated with the Cband rectifier for WiCoPT operation. The BPF extracts IF signals for data receipt. The center frequency of the BPF for $20 \mathrm{MHz}$ and $-3 \mathrm{~dB}$ bandwidth is $10 \mathrm{MHz}$. An FHX76LP GaAs HEMT (Sumitomo Electric Devices Inc.) is used. The substrate material is RO4350B (Rogers Co.). Center frequency of the rectifier is $5.80 \mathrm{GHz}$. The $5.8 \mathrm{GHz}$ rectifier is designed using the Advanced Design System (ADS) simulator (Agilent). The Angelov model [27], a nonlinear model of HEMTs, is used. Figure 5 shows simulated and measured load resistance characteristics of the RF-DC conversion efficiency under conditions of $20 \mathrm{dBm}$ input RF power and $5.80 \mathrm{GHz}$ frequency. A fabricated rectifier without BPF is used for the simulation and the measurement. The simulated and measured optimum load resistance is $439 \Omega$ and $470 \Omega$, since maximum conversion efficiencies of $53.3 \%$ and $49.7 \%$ are obtained. Figure 6 shows simulated and measured DC output power and rectification efficiency of the fabricated rectifier using a $5.80 \mathrm{GHz} \mathrm{CW}$ signal. The maximum conversion efficiency of $53.3 \%$ and the maximum DC output power of $328 \mathrm{~mW}$ are simulated for input power of $22 \mathrm{dBm}$ and $30 \mathrm{dBm}$, respectively. The maximum conversion efficiency of $49.7 \%$ and the maximum DC output power of $104 \mathrm{~mW}$ are measured for input power of $20 \mathrm{dBm}$ and $28 \mathrm{dBm}$, respectively. The BPF effect is negligibly small compared to the RFDC conversion characteristics. During measurements, the optimum load resistance of $470 \Omega$ is used. Conversion loss, defined as the power level difference between input RF and output IF signals, is measured. The conversion loss of $25.8 \mathrm{~dB}$ from $5.8 \mathrm{GHz}$ to $20 \mathrm{MHz}$ is obtained from the measurement while the input RF power level is $20 \mathrm{dBm}$.

A $5.8 \mathrm{GHz}$ rectifier for WiCoPT technology was thus successfully developed using the GaAs HEMT.

3.2. Evaluation of WiCoPT with Beam Steering. This section describes results of an experimental demonstration of a $5.80 \mathrm{GHz}$ WiCoPT system with beam steering capability. Figure 7 shows a block diagram of the demonstration. In the demonstration, configuration of a directional coupler is applied to show the validity of the configuration described in Section 2. The transmitting side has horizontal $1 \mathrm{D}$ beam steering capability using four phase shifters operating at $5.80 \mathrm{GHz}$. The phase shifter is a four-bit digital phase shifter [25]. A $4 \times 4$ circular patch array is used as four $1 \times 4$ subarrays. The $4 \times 4$ array is placed on a turntable so that the array beam is directed to the horn antenna when the beam is steered. The array antenna is designed using HFSS (Ansys Co.). Physical parameters such as patch diameter and element spacing are optimized by $3 \mathrm{D}$ electromagnetic field simulation. The phase shifter is controlled by PXI hardware (National Instruments Co.). The LabVIEW software individually controls DC biases 


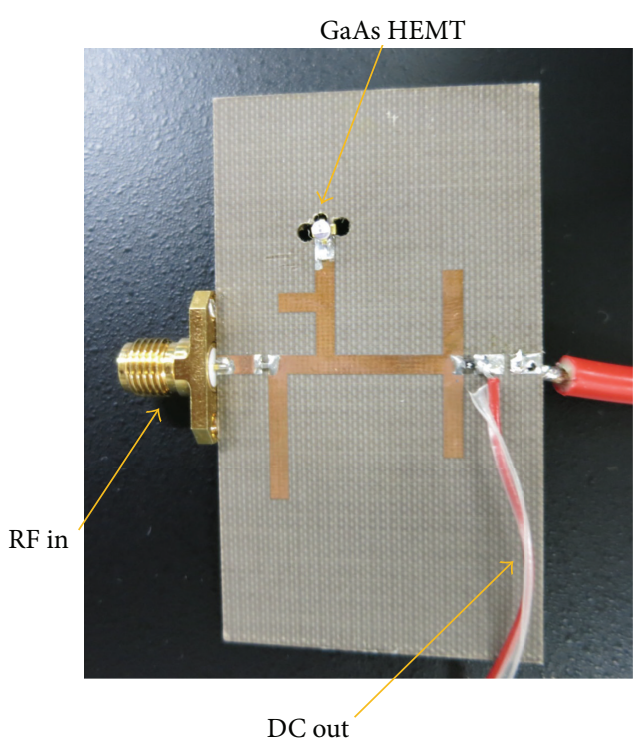

(a)

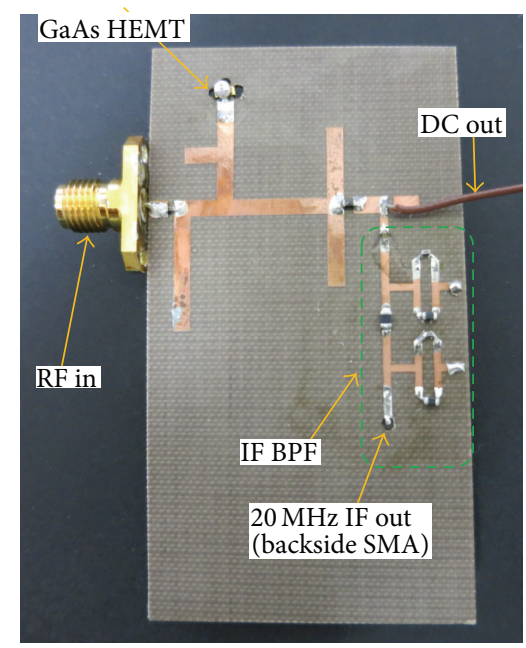

(b)

FIgURE 4: Fabricated C-band rectifier: (a) without BPF and (b) with BPF.

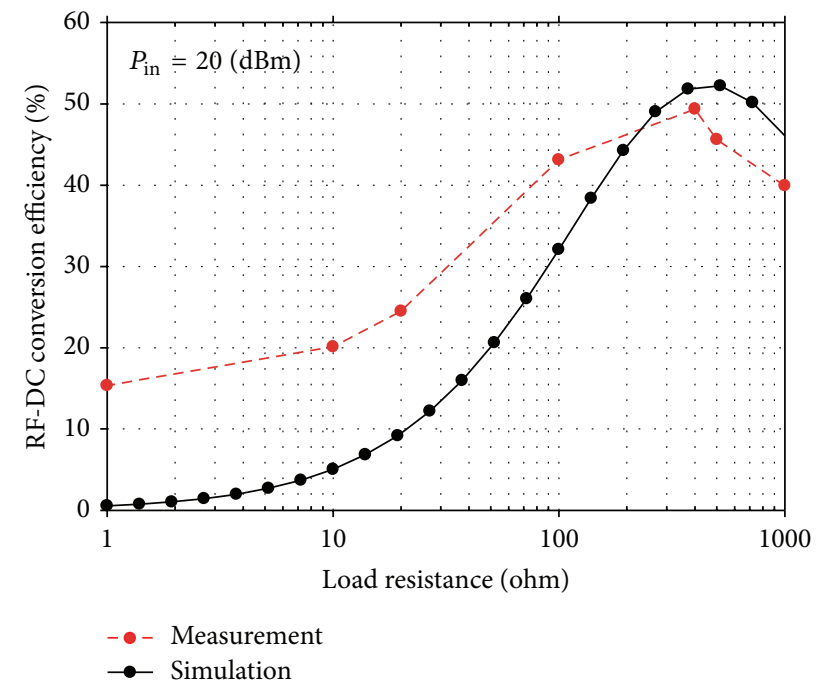

FIGURE 5: Simulated and measured load resistance characteristics of RF-DC conversion efficiency.

of the SPDT switches in the 4 phase shifters. The system can arbitrary create four-bit phase shifts, so beam direction is also arbitrary controlled digitally in the horizontal direction by adding phase shift between the element antennas.

The modulated baseband signal is upconverted to $5.80 \mathrm{GHz}$ and equally divided into four branches. The modulation method is quadrature phase shift keying (QPSK), and the sample rate is 1 MSPS. A pseudonoise $(\mathrm{PN})$ sequence of order 14 is used as random data. A root-raised cosine filter with alpha 0.5 is used in both the transmitting and receiving sides. The modulation/demodulation evaluation system of the PXI hardware is used. A $10 \mathrm{~dB}$ directional coupler is used

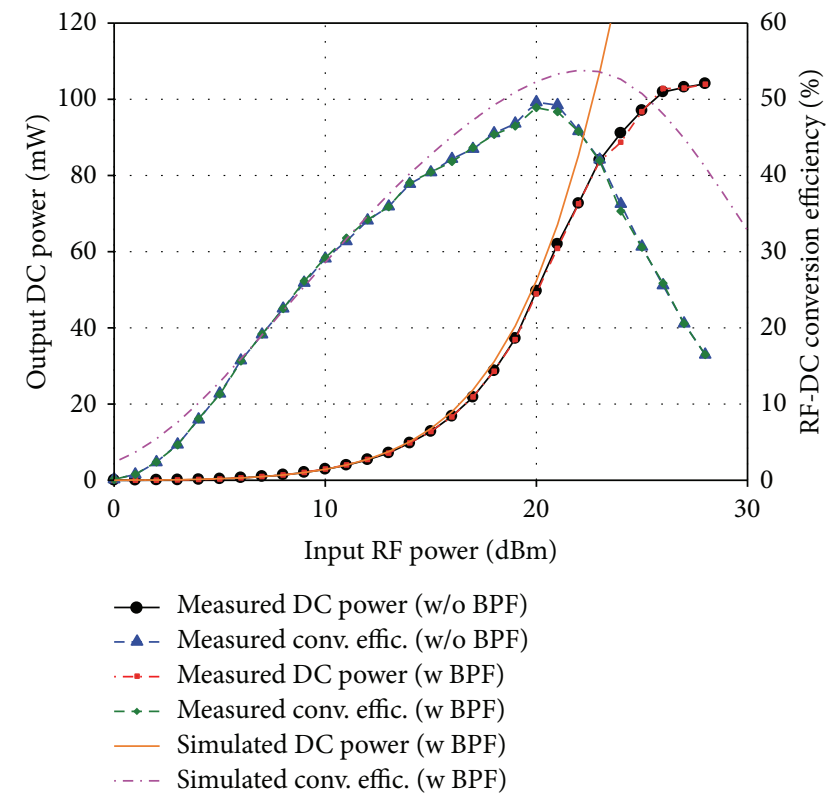

FIGURE 6: Simulated and measured DC output power and rectification efficiency of the fabricated rectifier using a $5.8 \mathrm{GHz} \mathrm{CW}$ signal.

in the demonstration to divide the received power for both the demodulation and rectification parts, so $90 \%$ power of the received signal is used for rectification. The rectifier without BPF described in Section 3.1 is used. After rectification, DC power is consumed by a load resistance of $470 \Omega$ and conversion efficiency is measured. Total output RF power of the transmitting side is $36 \mathrm{dBm}$ when the beam is centered. The distance between the antennas is $1 \mathrm{~m}$. Antennas on the transmitting and receiving side are $4 \times 4$ circular patch array and horn antennas, respectively. 


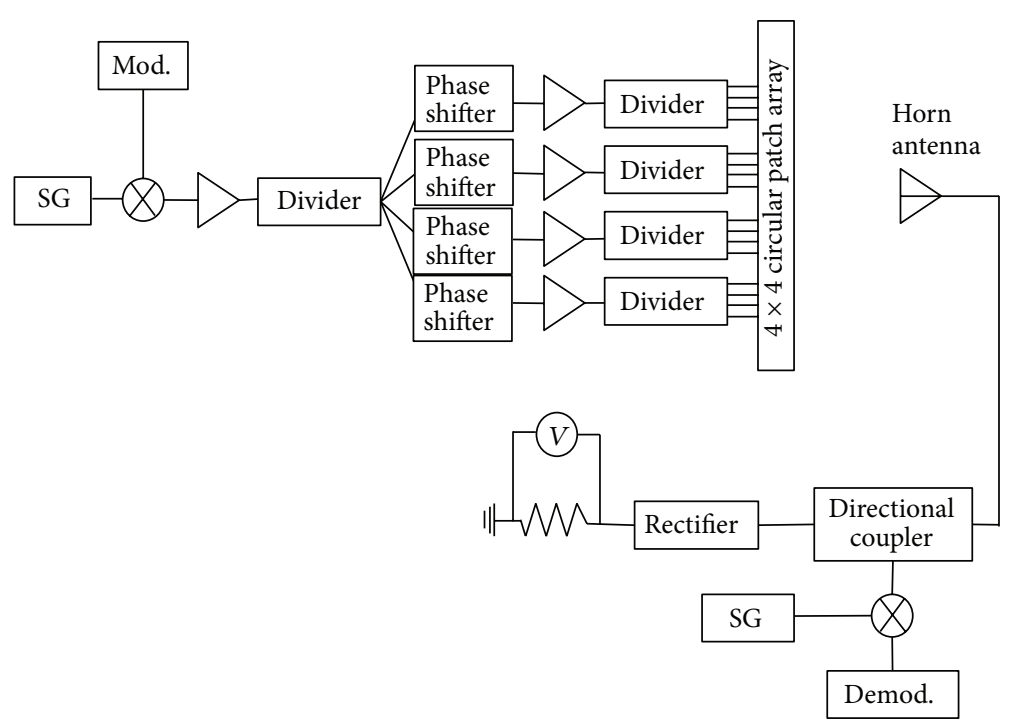

FIGURE 7: Block diagram of the experimental demonstration of the WiCoPT technology with beam steering.

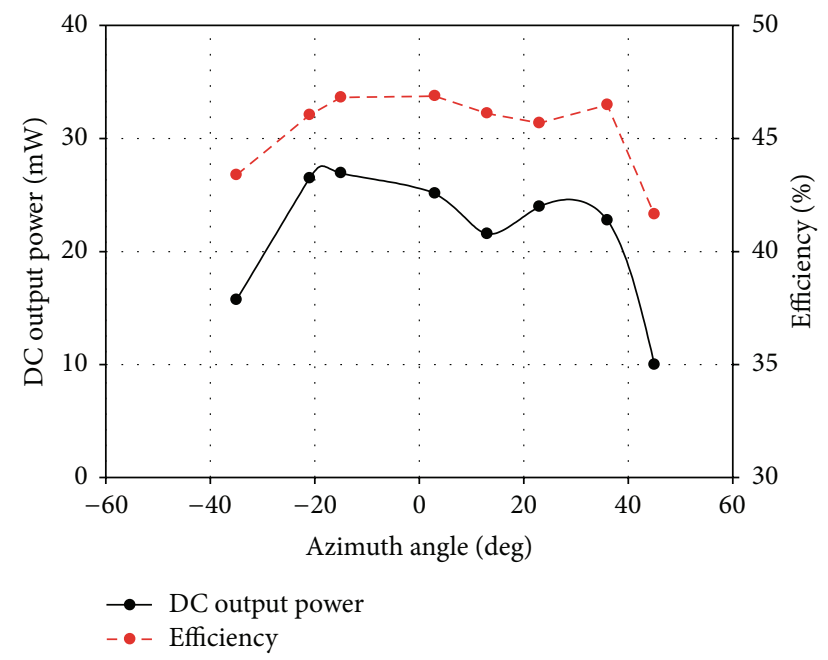

FIGURE 8: Measured RF-DC conversion efficiency and DC output power when the beam is steered.

Figure 8 shows the measured RF-DC conversion efficiency and DC output power when the beam is steered. Over $45 \%$ efficiency is obtained when the beam is steered from $-20^{\circ}$ to $35^{\circ}$. Figure 9 shows the constellation of the received signal. When the beam is steered to $+35^{\circ}$, the error vector magnitude (EVM) and bit-error rate (BER) of the received signal are $14.6 \%$ and below $6.1 \times 10^{-5}$, respectively. When the beam is steered to $-20^{\circ}, \mathrm{EVM}$ and BER of the received signal are $14.9 \%$ and below $6.1 \times 10^{-5}$, respectively. The measurement range of the BER is limited to $6.1 \times 10^{-5}$, due to the hardware memory.

The BER is affected by the coupling factor of the directional coupler. The coupling factor should be determined considering the balance between sensitivity of the receiver and rectification efficiency. In the demonstration shown in this section, a coupling factor of $10 \mathrm{~dB}$ is selected since communication quality is prioritized, such as BER of below $6.1 \times 10^{-5}$, which is the measurement limitation. The measured BER deteriorates to $3.2 \times 10^{-3}$ when a $20 \mathrm{~dB}$ directional coupler is used. Therefore, a coupling factor larger than $10 \mathrm{~dB}$ deteriorates the BER, as in the PXI system. From the viewpoint of rectification efficiency, the input RF power of the rectifier in Figure 7 is considered to be around $17 \mathrm{dBm}$ from Figures 6 and 8 . The efficiency will degrade when a coupler with a coupling factor of less than $10 \mathrm{~dB}$ is used. Considering this trade-off, a coupling factor of $10 \mathrm{~dB}$ is used.

The beam steering function is thus effectively integrated with the WiCoPT technology. Also, wireless communication and power transfer are conducted simultaneously.

3.3. Battery Charging Demonstration Using WiCoPT. Figure 10 shows a block diagram of the battery charging demonstration using the WiCoPT technology. The concept is based on the BPF configuration described in Section 2. To deliver both DC power and data from the transmitter to the receiver, $5.80 \mathrm{GHz} \mathrm{CW}$ and $5.82 \mathrm{GHz}$ modulated signals are generated on the transmitter side. To get high DC output power and charge the battery, eight rectifiers are arranged in parallel on the receiver side. The $5.80 \mathrm{GHz}$ rectifier is equipped with a $20 \mathrm{MHz}$ BPF. Eight output signals from the BPF in the rectifier are combined and input into the demodulator. DC out terminals of the rectifier are connected to the battery charge control unit. To prevent overcharging, the control unit limits the voltage and current to $3.9 \mathrm{~V}$ and $80 \mathrm{~mA}$. At the beginning of charging, the control unit limits the current to $80 \mathrm{~mA}$. If the output voltage reaches $3.9 \mathrm{~V}$, which is the charge finish voltage, the control unit stops current flow to the battery. Output voltage of the rectifier changes since the load resistance, which corresponds with input resistance of the control unit, changes. The modulation method is QPSK, and the sample rate is 1 MSPS. A root-raised cosine filter with 


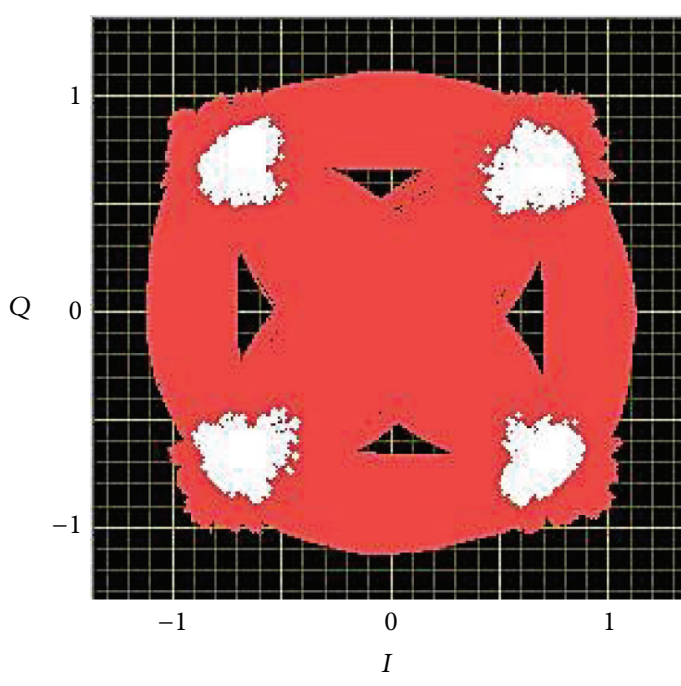

(a) +35 deg. beam steering

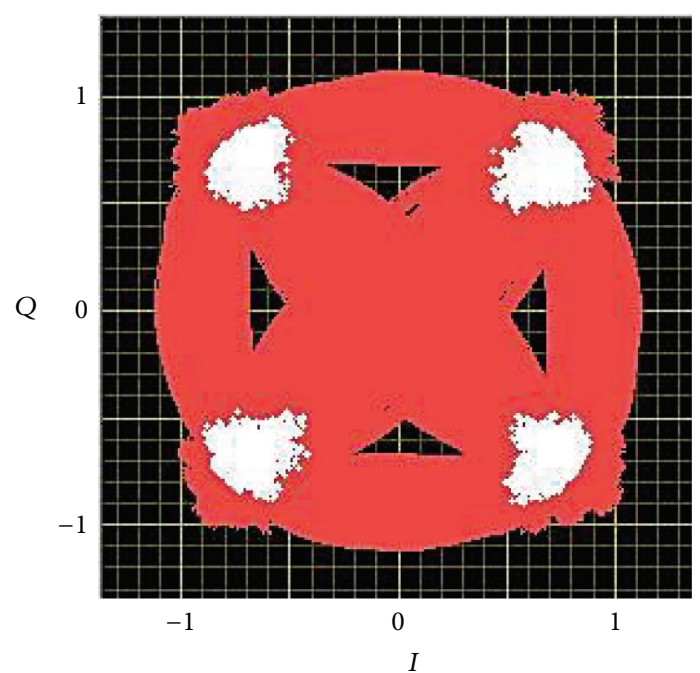

(b) -20 deg. beam steering

FIGURE 9: Measured constellations of the received IF signals when the beam is steered.

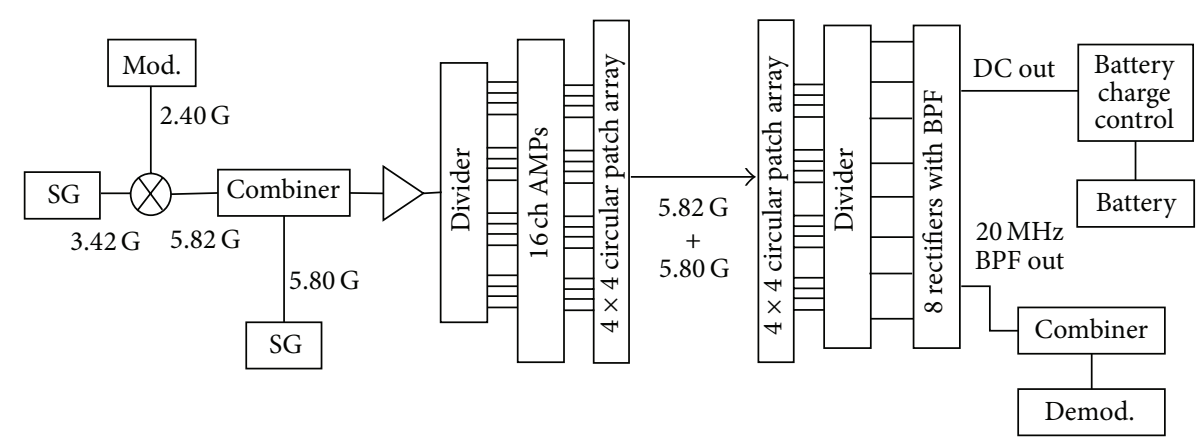

FIGURE 10: Block diagram of the battery charging demonstration using the WiCoPT technology.

alpha 0.5 is used on both the transmitting and receiving sides. The modulation/demodulation evaluation system of the PXI hardware is used. The distance between the transmitting and receiving antennas is $40 \mathrm{~cm}$. Total output power of the transmitter side is $40 \mathrm{dBm}$ and $31 \mathrm{dBm}$ at $5.80 \mathrm{GHz}$ and $5.82 \mathrm{GHz}$, respectively. Input power of the rectifier at $5.8 \mathrm{GHz}$ is calculated as $21.4 \mathrm{dBm}$ considering propagation loss, cable loss, gain of the amplifier, conversion loss of the mixer, and insertion loss of the combiner and divider.

The RF-DC conversion efficiency is measured by comparing the consumed power of the load and input RF power of the rectifier. EVM and BER are also measured. Measurement is carried out in an anechoic chamber. Figure 11 shows time domain characteristics of the voltage and current at the rectifier and the battery. It takes $20 \mathrm{~min}$ to charge the battery to $3.9 \mathrm{~V}$. The battery charge control unit has operated normally since charging current is initially limited to $80 \mathrm{~mA}$ and finally to $0 \mathrm{~mA}$. Battery charging capability is confirmed from the evaluation. Input resistance of the unit changes during charging, so an additional circuit will be required to obtain maximum RF-DC conversion efficiency in future applications.
Figure 12 shows constellations of the received signal. EVM and BER of $11.5 \%$ and below $6.1 \times 10^{-5}$ are measured, respectively. The BER measurement range is restricted to $6.1 \times$ $10^{-5}$ due to the hardware memory. We have thus validated the WiCoPT concept, including both communication and power functions.

We have confirmed the validity of the WiCoPT technology using the two configurations described in Sections 3.2 and 3.3 and shown that microwave band WiCoPT technology is effective for future integration in WPT-based communication systems.

\section{Application of WiSEnT in Completely Wireless Sensor Network}

As an application of the WiSEnT technology, a wireless thermal sensor is powered by WPT technology using a microwave band.

4.1. Overview of the Demonstration Using WiSEnT. Figure 13 shows a block diagram of a demonstration of the WiSEnT 


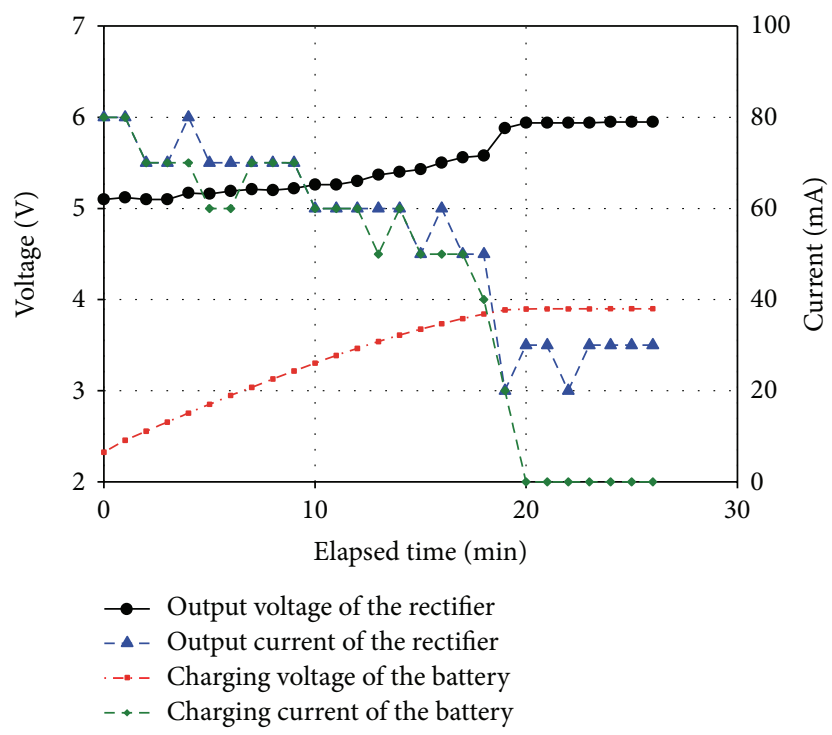

FIGURE 11: Time domain characteristics of voltage and current at the rectifier and the battery.

technology in a wireless thermal sensor. The base station and sensor node have WPT and communication functions. DC power consumed in the sensor node is supplied by WPT from the base station using an S-band CW signal. Thermal sensor data are then sent back to the base station using the $400 \mathrm{MHz}$ band. The distance between the base station and the sensor node is $1.2 \mathrm{~m}$. The signal source in the base station consists of a CW signal generator and an S-band high-power amplifier. The sensor node has an S-band rectifier, a thermal sensor, and a $400 \mathrm{MHz}$ band transceiver. DC power is provided by the S-band rectifier to the thermal sensor and transceiver. There is no battery in the sensor node. A $4 \times 4$ circular patch array antenna is used as both base station and sensor node antennas.

4.2. Design and Evaluation of S-Band Rectifier. One of the key devices of the technology is a microwave rectifier that can supply watt-class DC power. Figure 14 shows the S-band rectifier circuit. An NPT25015 GaN HEMT (Nitronex) is used for the rectifying element, since GaN HEMT has high breakdown characteristics comparable with GaAs ones, making the GaN device preferable for high-power application. Drain and source terminals are grounded to be used as Schottky diodes. A DC cut capacitor and an RF cut inductor are placed at the input and output terminals, respectively. Treatment of the harmonics is based on the design method described in Section 3.1. The design method shown in Section 3.1 is used.

Figure 15 shows simulated and measured results of the optimal load resistance under the conditions of $30 \mathrm{dBm}$ input RF power at $2.25 \mathrm{GHz}$. Optimal load resistance is $11 \Omega$, and the conversion efficiency is $10.8 \%$. Figure 16 shows simulated and measured input-output characteristics of the rectifier. A $2.25 \mathrm{GHz} \mathrm{CW}$ signal and $11 \Omega$ load resistance are used. A maximum RF-DC conversion efficiency of $36.7 \%$ is obtained when input power is $46 \mathrm{dBm}$. A maximum $\mathrm{DC}$ output power

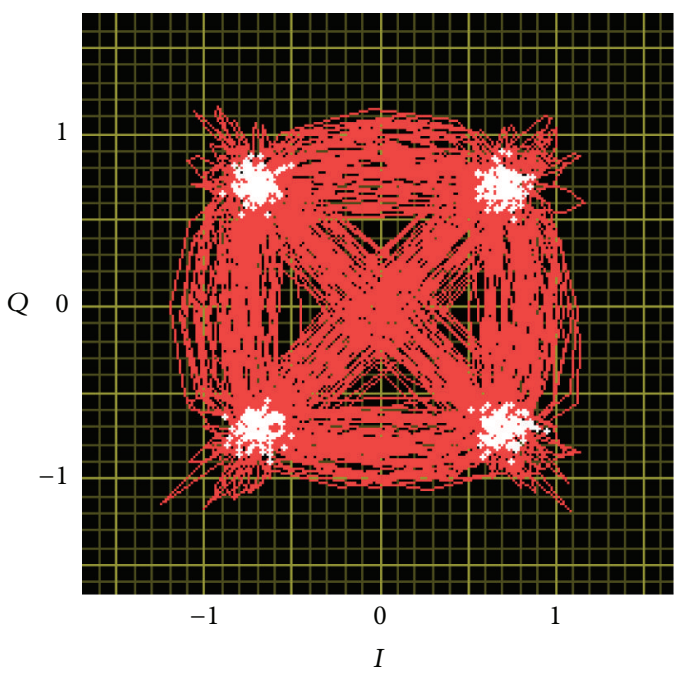

FIGURE 12: Constellations of the demodulated signal.

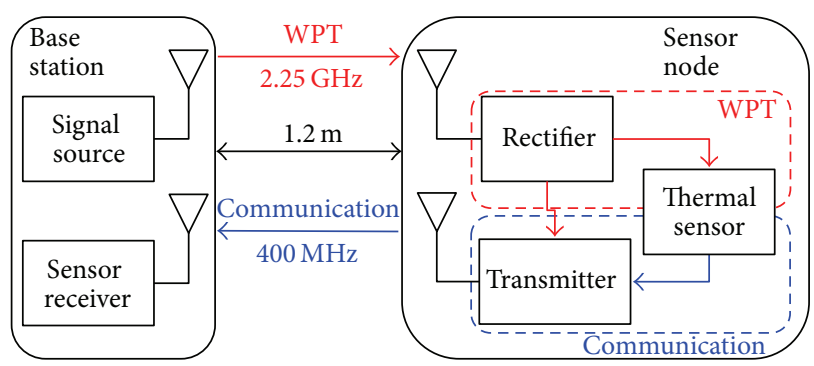

FIGURE 13: Block diagram of WiSEnT system using a wireless thermal sensor.

of $17.8 \mathrm{~W}$ is obtained when input power is $47 \mathrm{dBm}$. The measurement results agree well with the simulation results. We have thus successfully developed a high DC output power Sband rectifier for WiSEnT application.

4.3. Experimental Demonstration of WiSEnT. Table 3 shows a power diagram of the system. The total efficiency of conversion from RF power of the base station to DC power available in the sensor node is $0.35 \%$ when $47.6 \mathrm{dBm}$ output $\mathrm{RF}$ power from the base station is defined as the reference power. Input RF power of the sensor node is $35.6 \mathrm{~dB}$, which corresponds to $6.3 \%$ of the base station output power, considering $\mathrm{Tx} / \mathrm{Rx}$ antenna gain, feed loss, and propagation loss. RFDC conversion efficiency is calculated as $5.5 \%$ since power consumption of the thermal sensor of $200 \mathrm{~mW}$ and $\mathrm{RF}$ input power of $35.6 \mathrm{dBm}$ is considered. The RF-DC conversion efficiency is degraded since input resistance of the thermal sensor is not matched to the optimal load resistance of the rectifier. The total efficiency value is not pessimistically low since the total efficiency will be increased by adding more sensor nodes. At least a $100 \mathrm{~W}$-class high-power amplifier or high gain antenna will be required to maximize performance of the rectifier and exceed $10 \mathrm{~W}$ DC power. 


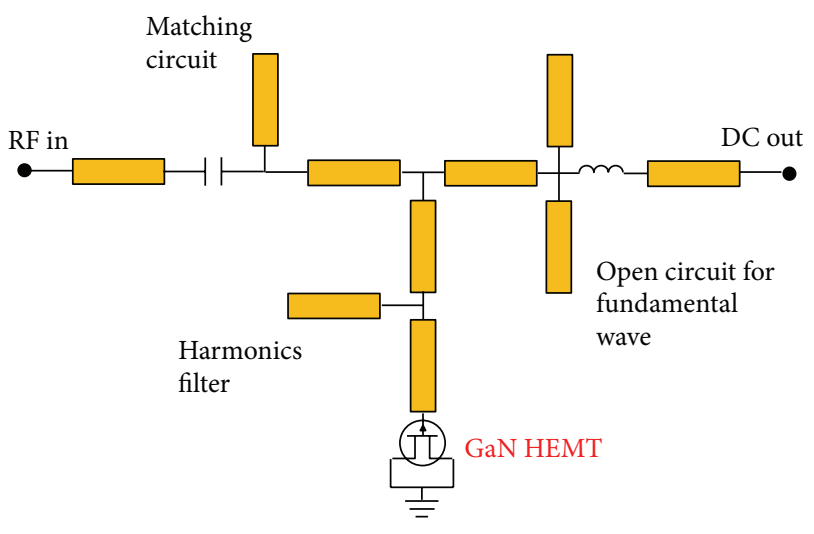

FIgURE 14: A circuit of the S-band high-power rectifier.

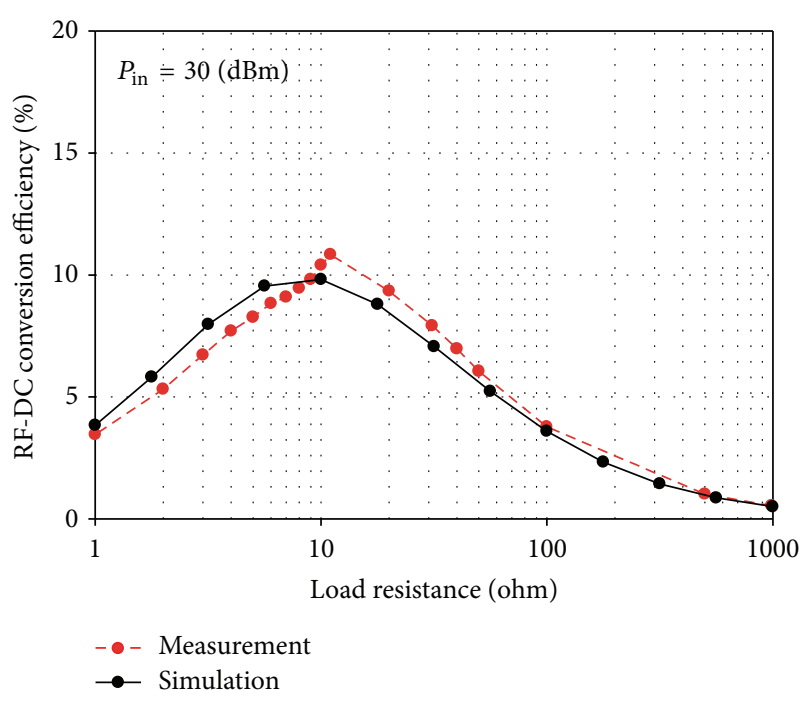

FIGURE 15: Simulated and measured results for optimal load resistance.

Figure 17 shows the measurement setup in an anechoic chamber. Two $4 \times 4$ arrays, the GaN rectifier, a thermal sensor, and ice for cooling the sensor are shown.

Figure 18 shows measured data from the thermal sensor, with sensor data and rectifier output voltage corresponding to the sensor supply voltage. In the demonstration, the thermal sensor is cooled by ice to show normal operation of the sensor. RF output is stopped and the sensor is cooled from 910 to 1035 s. At 1040 s, RF output and the sensor start. Sensor data show a temperature around $25^{\circ} \mathrm{C}$, which corresponds to room temperature. After the cooling, the sensor data show a temperature around $0^{\circ} \mathrm{C}$. Measurement of the system is conducted in an anechoic chamber. We have thus successfully demonstrated a completely wireless sensor network system using the WiSEnT technology.

\section{Conclusion}

We have proposed the WiCoPT and WiSEnT technologies and provided experimental demonstrations of the feasibility

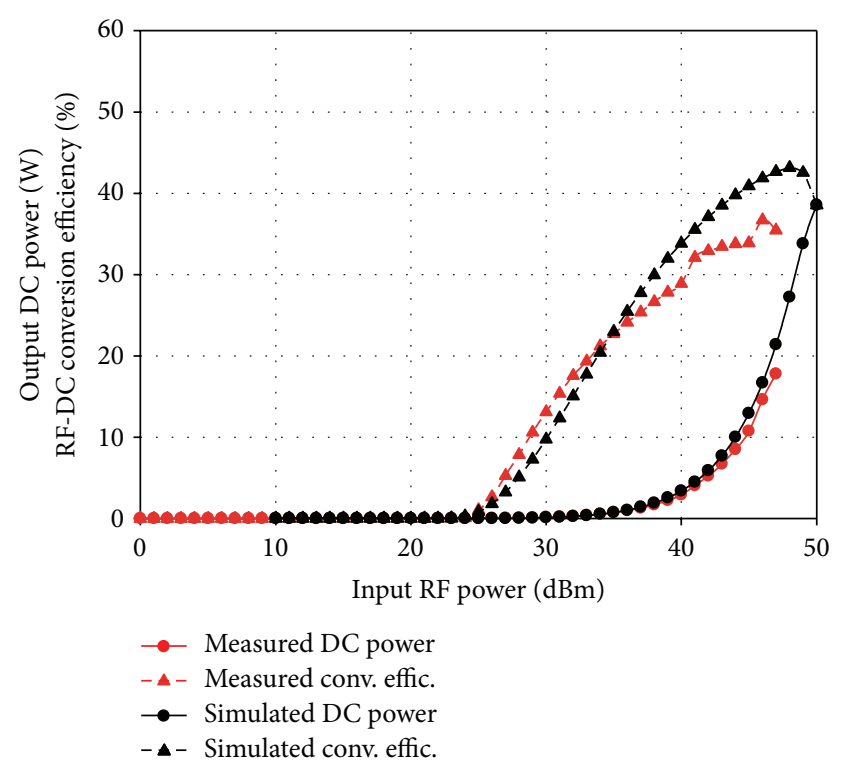

FIGURE 16: Simulated and measured input-output characteristics of the rectifier.

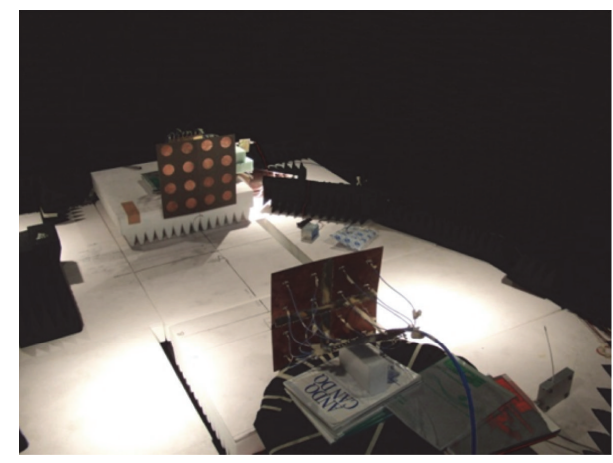

FIGURE 17: Measurement setup in an anechoic chamber.

for the coexistence of WPT and communication in a batteryfree wireless sensor network system. 5.80 GHz GaAs HEMT rectifiers with a $20 \mathrm{MHz} \mathrm{BPF}$ were designed and evaluated for WiCoPT technology. Output DC power was $50 \mathrm{~mW}$ with efficiency of $49.7 \%$. The fabricated rectifier was used for a WiCoPT demonstration. $400 \mathrm{~mW}$ output power was obtained and 1 MSPS QPSK communication with 11.5\% EVM was conducted. A high output power $2.25 \mathrm{GHz}$ GaN HEMT rectifier was designed for WiSEnT technology. A maximum output DC power of $17.8 \mathrm{~W}$ was measured. A battery-free wireless sensor network was created using the rectifier as a WiSEnT technology demonstration, and a wireless sensor network was realized by the WiSEnT technology. Our proposed technologies should be effective for a wireless power system integrated with communication functions in future wireless sensor network applications such as cars and spacecraft. 
TABLE 3: Power diagram for the demonstration.

\begin{tabular}{|c|c|c|c|c|}
\hline Amount of change at each term & & Power level (dBm) & Power level (W) & Total efficiency (\%) \\
\hline Signal source $(\mathrm{dBm})$ & 47.6 & 47.6 & 57.5 & 100 \\
\hline Feed loss $(\mathrm{dB})$ & 1.5 & 46.1 & & \\
\hline Tx antenna gain $(\mathrm{dBi})$ & 14.9 & 61.0 & & \\
\hline Propagation loss $(\mathrm{dB})$ & 39.1 & 21.9 & & \\
\hline $\mathrm{Rx}$ antenna gain $(\mathrm{dBi})$ & 14.0 & 35.9 & & \\
\hline Feed loss $(\mathrm{dB})$ & 0.3 & 35.6 & & \\
\hline Rectifier RF input (dBm) & - & 35.6 & 3.6 & 6.3 \\
\hline RF-DC conversion (\%) (rectifier) & 5.5 & - & - & \\
\hline $\begin{array}{l}\text { Supplied DC power }(\mathrm{dBm}) \text { (thermal } \\
\text { sensor) }\end{array}$ & - & 23.0 & 0.20 & 0.35 \\
\hline
\end{tabular}

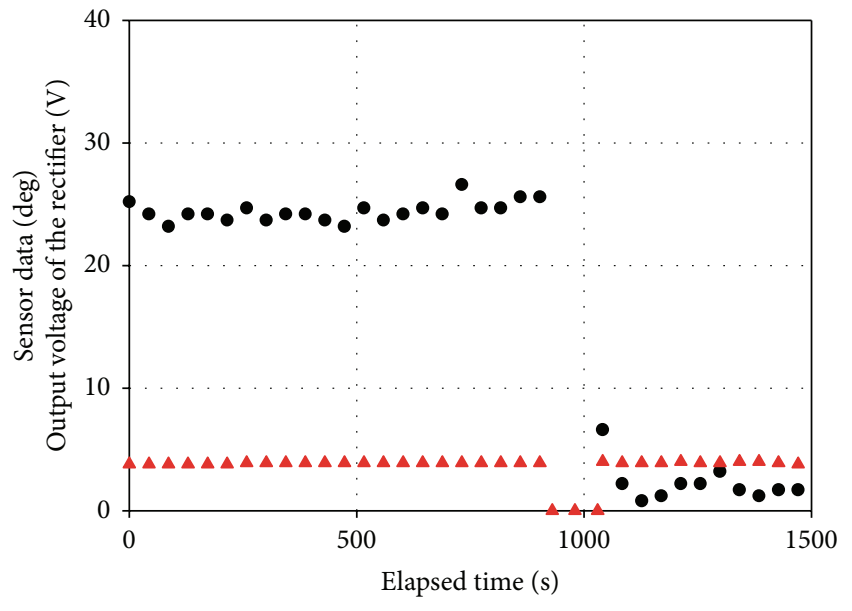

- Sensor data

- Output voltage of the rectifier

FIGURE 18: Measured data of the thermal sensor and output voltage of the rectifier.

\section{References}

[1] Y. Qingxin, Z. Xian, C. Haiyan, L. Yang, J. Liang, and Y. Rongee, "Direct field-circuit coupled analysis and corresponding experiments of electromagnetic resonant coupling system," IEEE Transactions on Magnetics, vol. 48, no. 11, pp. 3961-3964, 2012.

[2] D. W. Kim, Y. D. Chung, H. K. Kang, Y. S. Yoon, and T. K. Ko, "Characteristics of contactless power transfer for HTS coil based on electromagnetic resonance coupling," IEEE Transactions on Applied Superconductivity, vol. 22, no. 3, Article ID 5400604, 2012.

[3] T. C. Beh, M. Kato, T. Imura, S. Oh, and Y. Hori, "Automated impedance matching system for robust wireless power transfer via magnetic resonance coupling," IEEE Transactions on Industrial Electronics, vol. 60, no. 9, pp. 3689-3698, 2013.

[4] A. Kurs, A. Karalis, R. Moffatt, J. D. Joannopoulos, P. Fisher, and M. Soljačić, "Wireless power transfer via strongly coupled magnetic resonances," Science, vol. 317, no. 5834, pp. 83-86, 2007.

[5] N. Shinohara, "Wireless power transmission progress for electric vehicle in Japan," in Proceedings of the IEEE Radio and Wireless Symposium (RWS '13), pp. 109-111, Austin, Tex, USA.
[6] Z. Popovic, E. A. Falkenstein, D. Costinett, and R. Zane, "Lowpower far-field wireless powering for wireless sensors," Proceedings of the IEEE, vol. 101, no. 6, pp. 1397-1409, 2013.

[7] N. Oodachi, K. Ogawa, S. Obayashi, and H. Shoki, "Wireless power transfer system minimizing an influence of a desk," in Proceedings of the IEEE MTT-S International Microwave Workshop Series on Innovative Wireless Power Transmission, May 2012.

[8] X. Fang, H. Liu, G. Li, Q. Shao, and H. Li, "Wireless power transfer system for capsule endoscopy based on strongly coupled magnetic resonance theory," in Proceedings of the IEEE International Conference on Mechatronics and Automation (ICMA '11), pp. 232-236, August 2011.

[9] T. Salter, K. Choi, M. Peckerar, G. Metze, and N. Goldsman, "RF energy scavenging system utilising switched capacitor DC-DC converter," Electronics Letters, vol. 45, no. 7, pp. 374-376, 2009.

[10] E. Bonisoli, A. Canova, F. Freschi, S. Moos, M. Repetto, and S. Tornincasa, "Dynamic simulation of an electromechanical energy scavenging device," IEEE Transactions on Magnetics, vol. 46, no. 8, pp. 2856-2859, 2010.

[11] J. A. Paradiso and T. Starner, "Energy scavenging for mobile and wireless electronics," IEEE Pervasive Computing, vol. 4, no. 1, pp. 18-27, 2005.

[12] S. Keyrouz, H. J. Vissery, and A. G. Tijhuis, "Ambient RF energy harvesting from DTV stations," in Loughborough Antennas and Propagation Conference, November 2012.

[13] P. Nintanavongsa, U. Muncuk, D. R. Lewis, and K. R. Chowdhury, "Design optimization and implementation for RF energy harvesting circuits," IEEE Journal on Emerging and Selected Topics in Circuits and Systems, vol. 2, no. 1, pp. 24-33, 2012.

[14] N. Shinohara and T. Ishikawa, "High efficient beam forming with high efficient phased array for microwave power transmission," in Proceedings of the 13th International Conference on Electromagnetics in Advanced Applications (ICEAA '11), pp. 729732, September 2011.

[15] M. Oda, "Building a space solar power system," in Proceedings of the 2nd International Conference on Recent Advances in Space Technologies (RAST '05), pp. 24-27, June 2005.

[16] Y. J. Jang, Y. D. Ko, and S. Jeong, "Optimal design of the wireless charging electric vehicle," in IEEE International Electric Vehicle Conference (IEVC '12), March 2012.

[17] A. Hande, R. Bridgelall, and B. Zoghi, "Vibration energy harvesting for disaster asset monitoring using active RFID Tags," Proceedings of the IEEE, vol. 98, no. 9, pp. 1620-1628, 2010. 
[18] H.-J. Jung, S.-W. Lee, and D.-D. Jang, "Feasibility Study on a new energy harvesting electromagnetic device using aerodynamic instability," IEEE Transactions on Magnetics, vol. 45, no. 10, pp. 4376-4379, 2009.

[19] K.-J. Park, S.-M. Kang, H. S. Kim, C.-W. Baek, and T. K. Chung, "Energy scavenging system utilising MEMS switch for power management," Electronics Letters, vol. 48, no. 15, pp. 948-949, 2012.

[20] Y. Zhou, B. Froppier, and T. Razban, "Schottky diode rectifier for power harvesting application," in IEEE International Conference on RFID-Technologies and Applications, November 2012.

[21] H. Takhedmit, B. Merabet, L. Cirio et al., "A 2.45-GHz dualDiode RF-to-dc rectifier for rectenna applications," in Proceedings of the 13th European Microwave Conference, pp. 37-40, September 2010.

[22] M. Roberg, E. Falkenstein, and Z. Popovic, "High-efficiency harmonically-terminated rectifier for wireless powering applications," in IEEE International Microwave Symposium Digest, June 2012.

[23] S. Scorcioni, A. Bertacchini, L. Larcher, A. Ricciardi, D. Dondi, and P. Pavan, "RF to DC CMOS rectifier with high efficiency over a wide input power range for RFID applications," in IEEE International Microwave Symposium Digest, June 2012.

[24] K. Yamashita, T. Yamamoto, H. Seita, E. Shimane, K. Komurasaki, and S. Kawasaki, "An LTCC phase shifter with an SPDT switch for a $5.8 \mathrm{GHz}$-band active integrated phased array antenna," in Proceedings of the China-Japan Joint Microwave Conference (CJMW'08), pp. 447-449, September 2008.

[25] K. Yamashita, T. Yamamoto, H. Seita, E. Shimane, and S. Kawasaki, "A 16-element power AIPAA with a 4-bit digital LTCC phase shifter operating at the $5.8 \mathrm{GHz}$-band for wireless communication and power transmission," in Proceedings of the IEEE Radio and Wireless Symposium (RWW '10), pp. 308-311, January 2010.

[26] R. J. Gutmann and J. M. Borrego, "POWER COMBINING IN AN ARRAY OF MICROWAVE POWER RECTIFIERS," IEEE Transactions on Microwave Theory and Techniques, vol. 27, no. 12, pp. 958-968, 1979.

[27] I. Angelov, H. Zirath, and N. Rorsmann, "A new empirical nonlinear model for HEMT and MESFET devices," IEEE Transactions on Microwave Theory and Techniques, vol. 40, no. 12, pp. 2258-2266, 1992. 

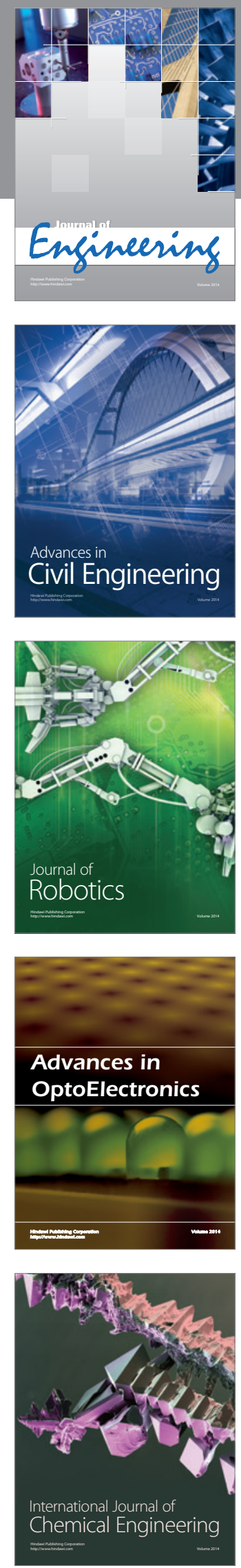

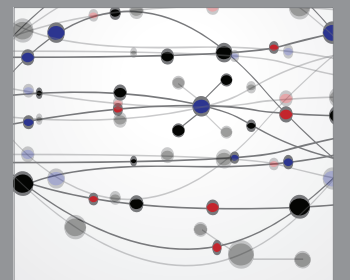

The Scientific World Journal
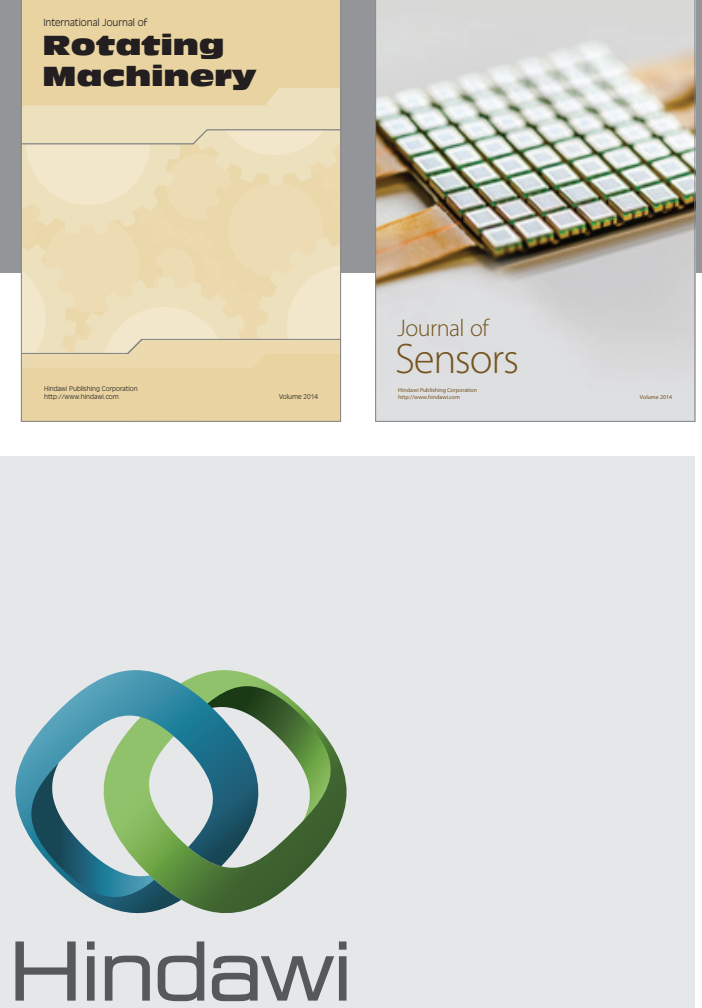

Submit your manuscripts at http://www.hindawi.com
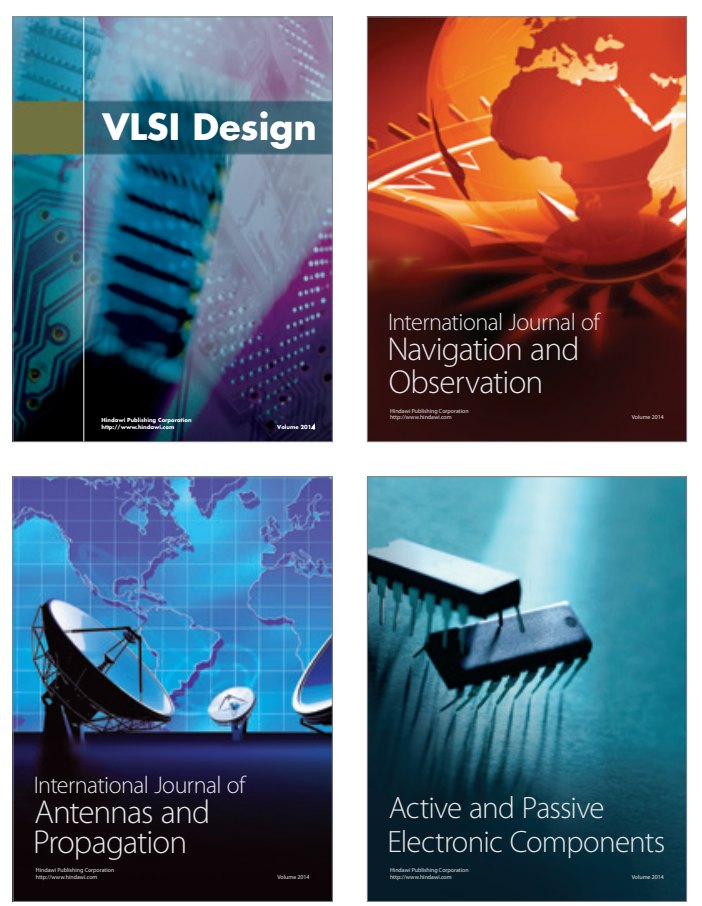
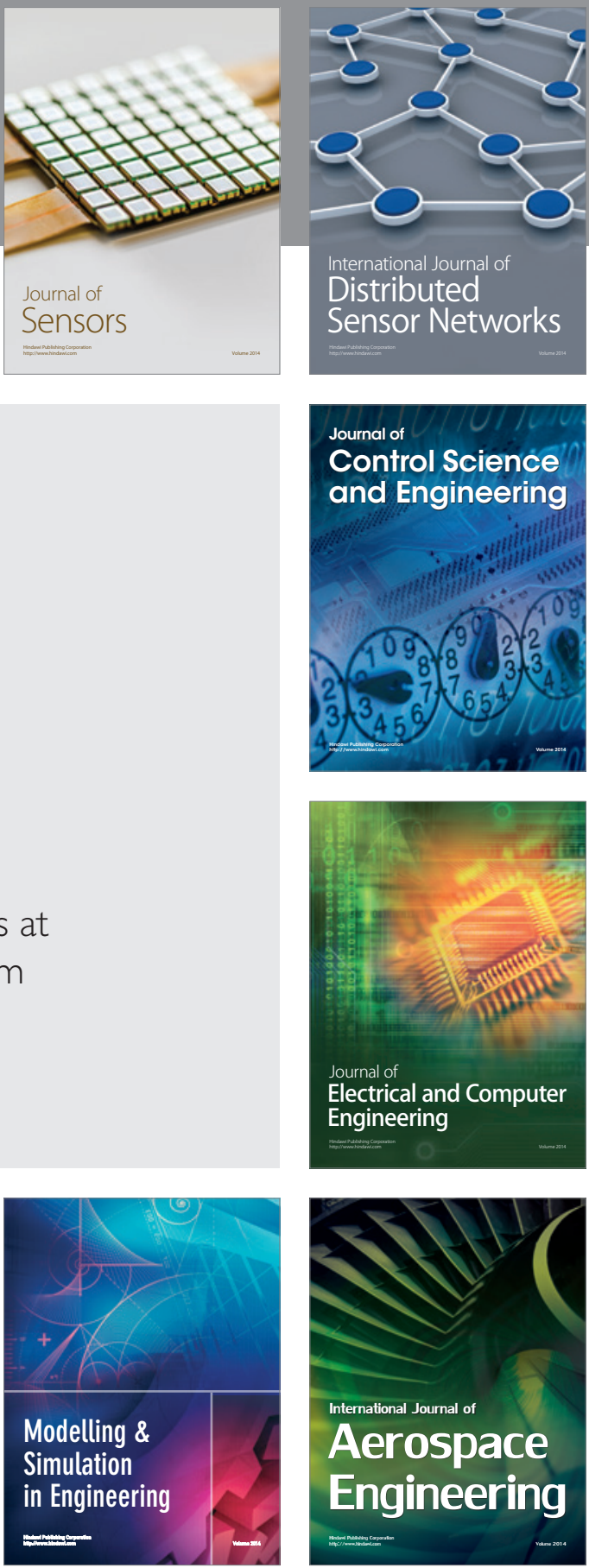

Journal of

Control Science

and Engineering
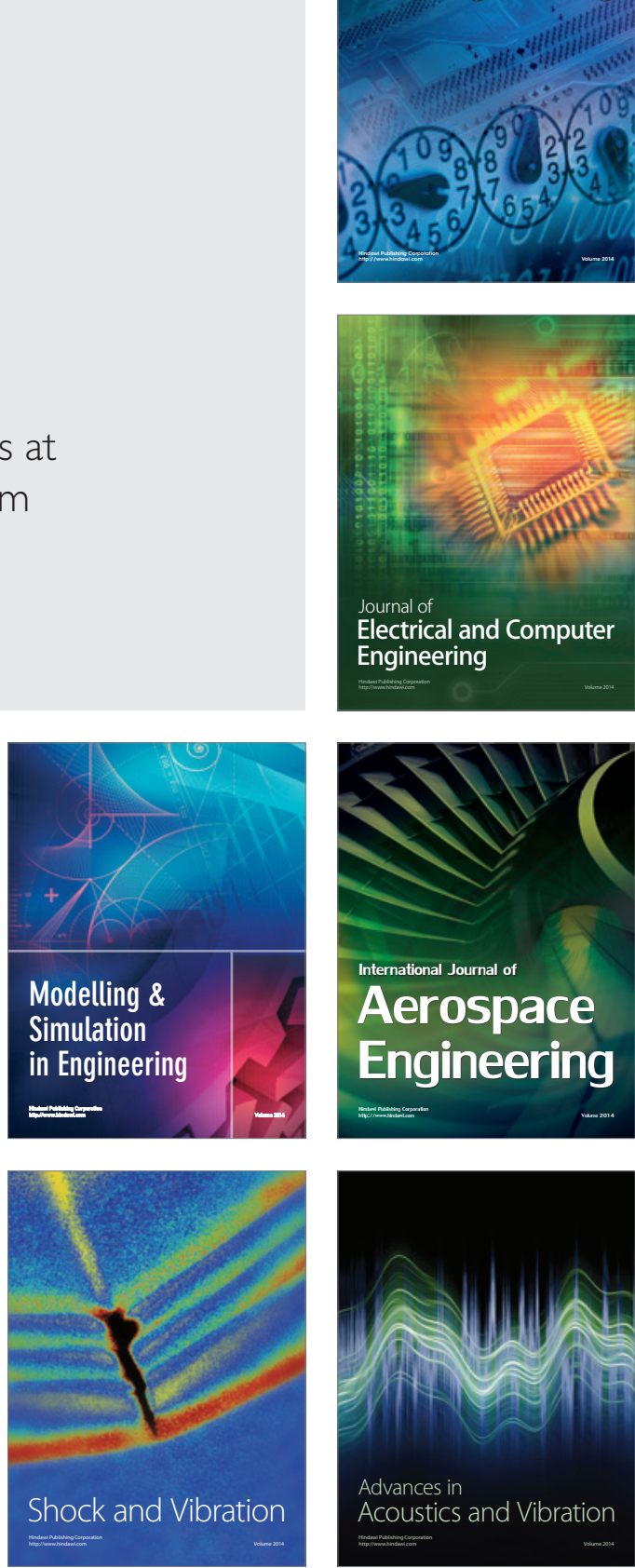